Sophie Visvikis-Siest*, Alex-Ander Aldasoro Arguinano, Maria Stathopoulou, Ting Xie, Alexandros Petrelis, Georges Weryha, Philippe Froguel, Peter Meier-Abt, Urs A. Meyer, Vid Mlakar, Marc Ansari, Andreas Papassotiropoulos, Georges Dedoussis, Baishen Pan, Roland P. Bühlmann, Mario Noyer-Weidner, Pierre-Yves Dietrich, Ron Van Schaik, Federico Innocenti, Winfried März, Lynn M. Bekris and Panos Deloukas

\title{
8th Santorini Conference: Systems medicine and personalized health and therapy, Santorini, Greece, 3-5 October 2016
}

DOI 10.1515/dmpt-2017-0011

Accepted for publication April 7, 2017; previously published online May 5, 2017

The 8th Santorini Conference was held in Santorini, Greece between the 3rd and 5th October 2016. It was realized in honor of Gérard Siest (link to video) (INSERM U1122; IGEPCV, University of Lorraine), the President of the seven previous Santorini Conferences, who passed away on 9 April 2016. As in the previous years, it was organized by the INSERM U1122; IGE-PCV (www.u1122.inserm.fr), University of Lorraine research group led by Sophie VisvikisSiest (Sofia Siest - President of the current and future Santorini Conferences).

*Corresponding author: Dr. Sophie Visvikis-Siest, INSERM UMR U1122; IGE-PCV, University of Lorraine, 30, rue Lionnois, 54000 Nancy, France, E-mail: sophie.visvikis-siest@inserm.fr Alex-Ander Aldasoro Arguinano, Maria Stathopoulou, Ting Xie, Alexandros Petrelis and Georges Weryha: INSERM UMR U1122; IGEPCV, University of Lorraine, Nancy, France

Philippe Froguel: Institut de Biologie de Lille, Lille, France Peter Meier-Abt, Urs A. Meyer and Andreas Papassotiropoulos: Biozentrum University, Basel, Switzerland

Vid Mlakar, Marc Ansari and Pierre-Yves Dietrich: Hôpital Universitaire, Genève, Switzerland

Georges Dedoussis: Harokopio University, Athens, Greece Baishen Pan: Medicine Zhongshan Hospital Fudan University, Shanghia, P.R. China

Roland P. Bühlmann: Bühlmann Laboratories AG, Schonenbuch, Switzerland

Mario Noyer-Weidner: Max Planck Institute for Molecular Genetics, Berlin, Germany

Ron Van Schaik: Erasmus MC Rotterdam, Rotterdam,

The Netherlands

Federico Innocenti: University of North Carolina, Chapel Hill, NC, USA Winfried März: SYNLAB Holding Deutschland GmbH, Augsburg, Germany

Lynn M. Bekris: Cleveland Clinic, Lerner Research Institute, Genomic Medicine, Cleveland, USA

Panos Deloukas: Queen Mary University of London, London, UK
The conference was held under the sponsorship of different international organizations (Gold: Bühlmann laboratories, Randox, Roche Diagnostics, ThermoFisher Scientific, Zinfandel Pharmaceutical - Silver: Siemens, Servier, Agena Bioscience, Synlab Akademie, DiaSys - Bronze: Metabolon, AB SCIEX-Others: Opusthree, Mastiha Growers Association), the BANQUE POPULAIRE and with the participation of: The International Federation of Clinical Chemistry and Laboratory Medicine (IFCC), the University of Lorraine, the Institut National de la Santé et de la Recherche Médicale (INSERM) and the European Commission. It was under the hospices of IFCC, European Federation of Clinical Chemistry and Laboratory Medicine (EFLM) and European Society of Pharmacogenomics and Theranostics (ESPT).

The scientific program consisted of nine sessions, one round table and five satellite meetings, realized in close collaboration with the ESPT and the H2020 Marie Skłodowska-Curie Actions (MSCA) - Research and Innovation Staff Exchange (RISE) - 2015 MAST4HEALTH Project (4 from ESPT working groups and 1 from MAST4HEALTH Project). In 5 specific poster sessions, 43 posters grouped in 2 thematics have been presented: Group A: Omics, environment and chronic diseases and Group B: Pharmacogenomics. Two 'Gérard Siest' awards have been given to the two best posters, one granted by the University of Lorraine and one by the IFCC. The conference also hosted the 3rd meeting of the VEGF Consortium (www.vegfconsortium.org), which aims to promote the research projects dedicated to the use of vascular endothelial growth factor (VEGF) '-omics' as stratified medicine's biomarkers of chronic diseases. Some 140 participants, coming from 33 countries from all around the world, had the opportunity to attend the presentations of 34 speakers on the following topics:

- First day: Systems Medicine and three specific pharmacogenomics and clinical prospective sessions 
- Second day: Phenotypes and environment effects, particularly nutrition, important for the study of physiological variations and chronic diseases

- Third day: Personalized therapy and the use of the new genomic biomarkers. The recent introduction of new drugs and development of biological pathways.

Here we present a summary of the presentations and the key messages of the 3 days of the conference.

\section{From systems biology to systems medicine}

Chairs: Sofia Siest, Nancy, France/Philippe Froguel, Lille, France

Sofia Siest (Nancy, France) had the first word to give an introduction to the 8th Santorini Conference. She gave a brief summary about how this colloquium has evolved in the last years, reminding that all these processes would not have been possible without the talent and hard work of Pr. Gerard Siest. Throughout 14 years, 8 "Santorini Conferences" welcomed a world of passion for science, specifically Personalized Medicine sessions on Genetics and Pharmacogenomics of risk factors and chronic diseases [1-4]. Over the years, the Santorini Conferences became one of the most important conferences on genetic predisposition to health, diseases, response to drugs and environment.

The debate about how important it is to develop a personalized treatment in which therapy is optimized and costs controlled was held by Faiez Zannad (Nancy, France) using heart failure as an example of diseases. The standard medical treatment for heart failure is complex and there is an increasing interest in detecting cardiovascular biomarkers for a better clinical application. He described the HOMAGE project, an EU FP7 program (http://www.homage-hf.eu/) [5] where the goal is to identify omics-based biomarkers that can detect pathological processes predictive of the development of heart failure to allow early mechanistically driven therapeutic interventions for preventing heart failure.

Finishing with the systems biology to systems medicine session, we had Andreas Papassotiropoulos (Basel, Switzerland). He introduced us into the wide field of epigenetic modifications and more specifically, into the DNA methylation undergoing with age [6]. Methylation regulates imprinting, chromosomal inactivation and gene expression. The existing results show that age is the most potent factor correlated with the global DNA methylation. These data can be used to identify the hidden molecular mechanisms of age-related traits relevant to health and disease. Interestingly, age-related methylation loci are within regulatory regions of genes closely related with Alzheimer's disease (AD) and cancer. Thus, the decomposition of blood methylome-wide patterns bears considerable potential for the study of brain-related physiological and pathological traits.

\section{Systems pharmacogenomics and mechanisms of drug action}

Chairs: Peter Meier-Abt, Basel, Switzerland/Urs A. Meyer, Basel, Switzerland

John Ryals (Metabolon, Raleigh, USA) reminded us that to be able to advance in precision medicine, it is essential to identify response biomarkers and to precisely define drug action. Although gene sequencing has yielded massive quantities of data, which have resulted in many important insights, it has been difficult to discern actionable signals from it. One way to go through the complexity of the data is by using metabolomics, able to precisely measure 2000 molecules in plasma. Metabolomics provides a real-time assessment of the phenotype, helps to unravel complex traits and to detect meaningful signals within the genome [7]. Novel technological innovations enable metabolomics to deliver signatures of disease and provide an integral tool for assessing mechanisms of drug action, thus expanding the boundaries of precision medicine and systems pharmacogenomics.

Pablo Villoslada (Barcelona, Spain) followed with a presentation about multiple sclerosis (MS), a complex disease where the effect that the combined therapies will have at the clinical level is difficult to predict [8]. In order to predict the effect of combination therapies, the group of Pablo Villoslada has developed the CombiMS EU project, a systems medicine approach to (i) characterize the signaling pathways that mediate MS and (ii) predict new combination therapies based on logic networks simulations. Measurements of the phosphorylation of key proteins involved in MS in 150 patients and 50 controls were analyzed. These phospho-proteins were used to identify the single active network that best fitted the data for each patient. Finally, an approach was proposed for predicting combination therapy based on network topology. All this work can be used for developing combination therapies for other complex diseases.

Continuing within the drug actions field, Federico Innocenti (Chapel Hill, NC, USA) discussed the efficacy and toxicity of VEGF inhibitors in cancer patients [9]. 
Approved in 2004, the first anti-VEGF drug, bevacizumab, is widely used in combination with chemotherapy in a large variety of tumor types. Despite the survival advantage, its use still faces a number of barriers, including significant toxic side effects. The failure to identify clinically useful biomarkers that can consistently predict clinical efficacy of this agent and its safety profile has been a significant hurdle for the use of bevacizumab. It is important to make predictive tools available to physicians to identify those patients who are unlikely to benefit from bevacizumab so that the associated toxicities could be also avoided. Dr. Innocenti has presented studies using germline genomics in a large series of cancer patients to discover the genetic determinants of efficacy and toxicity of VEGF inhibitors.

\section{Pharmacogenomics, immunotherapy and onco-hematology}

Chairs: Marc Ansari, Geneva, Switzerland/Maja Krajinovic, Montreal, Canada

Marc Ansari (Geneva, Switzerland) opened the session with the talk on Hematopoietic Stem Cell Transplantation (HSCT) for pediatric patients. He began the lecture by introducing basic data and overview of transplantation. Next, basic decision-making process before transplantation and presentation of data on survival were discussed in order to present the problematics of side-effects such as veno-oclusive disease, graft versus host disease, hemorrhagic cystitis and infection associated with the drugs (busulfan and cyclophosphamide) used for conditioning regimen. The lecture continued with the presentation of busulfan and clinical data on mortality and frequency of adverse side-effects followed by presentation of biochemistry and usage of candidate gene approach to identify association between glutathione transferases genes (GSTs) and toxicity, mortality and busulfan [10]. A detailed presentation of the results obtained on glutathione S-transferase Mu 1 (GSTM1), glutathione S-transferase P (GSTP1) and glutathione S-transferase A1 (GSTA1) genes using in vitro approach as well as gene association studies was given. The lecture closed by discussing the importance of replication and the need of prospective randomized trials. Professor Ansari presented the efforts taken in order to validate current results. He presented on behalf of European Group for Blood and Marrow Transplantation (EBMT) and ESPT an outline of a large ongoing prospective trial for children with an acute lymphoblastic leukemia receiving a HSCT that involves more than 20 countries with different ethnicity to validate the results.

The second speaker of the session was Pierre-Yves Dietrich (Geneva, Switzerland). In the beginning of his talk, he outlined the evidence for immune system involvement in the development of cancer, followed by a review of current understanding of T-cell biology and how these cells mediate the killing of cancer cells [11, 12]. Next, he outlined current understanding of cancer defense mechanism against immune system through inhibition of interactions, excretion of soluble mediators, modulation of cell response and significant changes of local microenvironment, resulting in suppression or modulation of the immune system activity. The lecture continued with detailed discussion of different options for immunotherapy against cancer: therapeutic vaccines, chimeric antigen receptors (CAR) T-cells, inductions of changes to tumor environment and immune-editing. During the lecture special weightage was given to CAR T-cells and different generations of their development with focus on advantages and problematic aspects that need further attention before implementation in clinical practice. The talk was concluded with discussion of other immune system modulators already used in clinical practice or under investigation in clinical trials.

Aurore Perrot (Nancy, France) presented a talk on multiple myeloma targets [13]. She began her talk by reviewing the clinical features of multiple myeloma. She continued with the discussion of oncogenesis and most frequent genetic changes occurring during the development of multiple myeloma. Next, she outlined and described in detail current major targets for drugs: proteasome, surface/receptor molecules, modulation of immune system, epigenetic modulation of oncogene or tumorsuppressor gene and immune checkpoint inhibitors. Most extensive discussion was dedicated to drugs currently used in clinics or being tested in clinical trials with special focus on daratumumab, elotuzumab, histone deacetylase (HDAC) inhibitors and nivolumab. She concluded by stressing the importance of genetic abnormalities associated with the choice of drug used for treatment and the challenges for research in the future.

Maja Krajinovic (Montreal, Canada), presented a lecture on pharmacogenomics of leukemia [14]. During the introduction, she presented general overview of leukemia, its treatment, problems associated with short- and long-term toxicities and differences in individual response to the drugs used. The talk continued with the presentation of a study where associations between methotrexate, thymidylate synthase, relapse and events-free survival were identified. The lecture continued with presentation 
of next-generation sequencing technology and opportunities in the field of pharmacogenetics and presentation of her group's recent results. During the second part of the talk, genes associated with asparaginase treatment (MYBBP1A) and allergies, pancreatitis and thrombosis were discussed. Next, results on IL16 association with pancreatitis and BAHD1 association with vincristine, events-free survival and neurotoxicity were presented. The lecture concluded with a discussion of future direction where special attention to basic functional and in vivo studies should be given followed by retrospective and prospective clinical trial.

\section{Genomics and proteomics of Alzhei- mer's disease}

Chairs: Andreas Papassotiropoulos, Basel, Switzerland

The first presentation was given by Lynn M. Bekris (Cleveland, USA) about genetic variations within the apolipoprotein E (APOE) locus and their associations with late-onset $\mathrm{AD}$ risk $[15,16]$. To explore whether $A P O E$ locus cis-regulatory elements might contribute to regional gene regulation, Bekris et al. produced regulatory region reporter constructs containing haplotypes of $A P O E$ locus promoters for APOE, APOC1 and TOMM4O as well as for other potential enhancers. Results demonstrate that multiple $A P O E$ locus cis-elements influence both $A P O E$ and TOMM40 promoter activity, suggesting a complex regulatory structure. These results have important implications for $\mathrm{AD}$ therapeutic strategies that focus on targeting $A P O E$ expression.

Erich E. Wanker (Berlin, Germany) continued the session with a presentation on the protein misfolding diseases such as AD, Parkinson's disease and Huntington's disease, all of them characterized by the accumulation of insoluble protein aggregates in patient's brain. In order to better understand the exact nature of pathogenic aggregates and their mechanisms of toxicity in cells, Wanker et al. have developed a large interactome network, where they added multiple proteins involved in different neurodegenerative diseases $[17,18]$. The use of this network in combination with various OMICs approaches, aid them to identify "neurodegenerative disease modules." These disease modules highlighted proteins that are abnormally aggregated in brains of AD patients, suggesting that interactome maps are valuable resources, which enable the elucidation of common disease mechanisms.

Continuing with the AD, Ellen Umlauf (Vienna, Austria) presented a case-control study, which included healthy individuals, patients with mild cognitive impairment (MCI) and late-onset AD patients. The goal of this study was to identify additional single nucleotide polymorphisms (SNP) candidates associated with MCI. The results obtained after an additive logistic regression showed novel genetic markers linked to the MCI [19]. They also emphasized the importance of carefully characterized controls in addition to well-diagnosed patients in case-control studies.

\section{Omics studies of human phenotypes and environment}

Chairs: Georges Dedoussis, Athens, Greece/Baishen Pan, Shanghai, China

Panos Deloukas (London, UK) focused on the genome-wide association studies (GWAS), which have identified several associations for coronary artery disease (CAD) explaining $15 \%$ of its heritability. Most of these signals are driven by common variants with low-effect associations. However, such variants seem to contribute minimally to the genetic architecture of CAD. The UK Biobank was established to improve understanding of the causes of common diseases including CAD and has completed the recruitment of 502,713 individuals [20]. Based on their NHS records and national registries, they identified 10,801 CAD cases and undertook an association analysis. Based on the initial results, there are several new CAD risk loci, which are, however, once again driven by common variants.

Robert Barouki (Paris, France), described the concept of exposome, which aims to integrate all environmental exposures over the life time [21]. This ambitious concept aims to be complemented with genome approaches. There are several different exposome projects, each one focused in different exposure fields, such as air pollution effects, water contaminants, early life exposure, etc. The different exposome projects in Europe and in the United states were briefly discussed.

Amalia Gastaldelli (Pisa, Italy) presented the importance of the interaction between the phenotype and environment in the non-alcoholic fatty liver disease (NAFLD) [22]. NAFLD is a metabolic disease, often associated with hepatic and systemic inflammation, insulin resistance and obesity. The -omics techniques including genomics, transcriptomics, epigenomics, metabolomics, exposomics and foodomics are currently used in clinical trials to identify mechanisms and risk factors.

Closing the session, Laurent Becquemont (Le Kremlin Bicêtre, France) made an introduction on the 
human plasma-metabolome. This is an interesting project including 800 healthy volunteers, where the aim is to define the "normal" levels of 185 targeted plasma metabolites [23]. They identified total blood cholesterol, gender and age as the main components explaining the variability of the studied human metabolome. This study provides an essential baseline to define the normal metabolome profile and the main sources of variation.

\section{Nutrition and metabolic health}

Chairs: Roland P. Bühlmann, Basel, Switzerland/Mario Noyer-Weidner, Berlin, Germany

The introduction to this session was made by Philippe Froguel (Lille, France), who spoke about obesity, a genetic trait with $70 \%$ heritability [24]. GWAS have identified more than 100 loci increasing BMI and mutations in genes mainly part of the regulation of food intake have been found to cause monogenic obesity. There are evidences that different parts of the brain are highly expressing obesity genes, suggesting a role in food intake behavior. Genes involved in nutrient processing and/or on gut microbiote composition are also involved in obesity and metabolism. Finally, system biology approaches suggest that -omics data may help predict effects of nutrients on metabolism, thus opening new directions towards personalized nutrition.

In his lecture, George Dedoussis (Athens, Greece) presented a different approach for the management of NAFLD. Instead of using pharmacological means for managing the disease, a dietary regimen with bioactive phytochemicals from fruits, vegetables and plants or their products was proposed [25]. Fifty five patients with NAFLD were enrolled in a two isocaloric dietary treatments for 24 weeks. Anthropometric, NAFLD Fibrosis score and biochemical tests were conducted pre- and post-intervention. The results showed a significant improvement of the patients enrolled in the study. He also described the MAST4HEALTH program, which aims to explore the effect of Mastiha, a natural product of Greece, which was shown to possess antioxidant/anti-inflammatory and lipid lowering properties.

\section{Pharmacogenomics and personalized/stratified therapy}

Chairs: Pierre-Yves Dietrich, Geneva, Switzerland/Ron Van Schaik, Rotterdam, The Netherlands
Maurizio Simmaco (Rome, Italy) started the session by presenting a novel predictive tool for $5 \mathrm{FU}$ drug toxicity and efficacy (5-fluorouracil) [26]. This chemotherapeutic agent is widely used and life-threatening side-effects can arise in as high as $30 \%$ of the patients. One of the factors affecting the toxicity is the dihydropyrimidine dehydrogenase (DPYD) gene polymorphisms, associated with low activity of the DPD enzyme. Although these polymorphisms are accepted as a good predictive test to recognize patient at risk of severe toxicity, they can identify only a small fraction of those patients. Because of this, the ex-vivo assay, called 5FUDR, has been developed, which can identify an increased fraction of patients who developed severe toxicity compared to DPYD genotyping.

Markus Paulmichl (Salzburg, Austria) discussed the difficulties of the implementation of pharmacogenomics moving from "diagnose and treat" to "predict and pre-empt" [27]. The implementation conundrum was presented and steps for solutions of the difficulties were proposed including amelioration of knowledge and dealing with analytical challenges. Examples of analytical challenges were presented for CYP2D6 and for the development of anticoagulant prescription.

Michael Marschler (Prahealthsciences, Mannheim, Germany), in his lecture introduced us on the influence of pharmacogenomics on pharmacovigilance activities (not published results). Due to gene-environment interactions there exists large variability in responses to drug therapy. Thus, serious adverse drug reactions can happen in specific sub-populations who may have different sensitivity to medicinal products. Because of this, for the preparation of risk management plans it is essential to consider the potential risk of genomic variations and identify risk minimizations measures.

Robin Everts (San Diego, USA) did an introduction on the current pharmacogenomics research on the absorption, distribution, metabolism and excretion properties of drugs. Although the research in this field is increasing rapidly, the pharmacogenetics-related allele's standardization is lacking. The presentation was focused on the contribution that Agena Bioscience can provide through examples of participation in the Genetic Testing Reference Materials Coordination Program [28].

To finish the session, Tiago Nava (Montréal, Canada) gave a presentation about the study of GSTA1 genetic variants and its importance in the evaluation of busulfan $(\mathrm{Bu})$ first dose in children [29]. The busulfan is a key component of conditioning before HSCT in children. They aim to evaluate the role of GSTA1 genotyping on performance of different models in predicting Bu first dose. For this, they enrolled 129 patients who underwent HSCT after 
$\mathrm{Bu}$ containing conditioning was included. They demonstrated that GSTA1 haplotypes clearly interfere in performance of dose prediction models in children, proposing that the development of new population PK study including GSTA1 haplotypes are necessary to a more evenly distributed exposure among pediatric patients.

\section{Genomic biomarkers and management of metabolic and inflammatory diseases}

Chairs: Federico Innocenti, Chapel Hill, USA/Winfried Marz, Augsburg, Germany

Behrooz Z. Alizadeh (Groningen, The Netherlands) started the session with a presentation on C-reactive protein, as an example of biomarkers with causal associations and he argued on its importance in classification of individuals into homogenous groups for diseases/risk factors (not published results). These results are essential in population-based medicine, where careful assessment of the validity and causality of biomarkers becomes important, when critical decision beyond prediction for an individual or a patient, intervention and management will be made.

M. Pilar Francino (Valencia, Spain) continued the session with a presentation about the microbial groups present in the human gut and their contribution to several basic physiological functions, including nutrition, defense against pathogens and metabolic and immune homeostasis [30]. Consequently, disturbances in the microbiota can result in several metabolic health problems. During this presentation, M. Pilar Francino reviewed some of the complex relationships between gut microbiota alterations and metabolic health. She showed us the high importance that the microbiota has in the regulation of host metabolism, mainly in relation to energy homeostasis and adiposity. Thus, concluding that personalized medicine will need to take into account the composition and function of an individual's gut microbiota in order to better prevent, diagnose and treat metabolic disorders.

Steffen Gay (Zurich, Switzerland) presented the field of epigenetic modifications in inflammation in general and in particular in rheumatoid arthritis [31]. The interplay of epigenetics is best illustrated by the involvement of multiple regulatory biological processes, such as acetylation, methylation, phosphorylation, sumoylation and noncoding RNAs, including microRNAs (miR) and long noncoding RNAs (lncRNA). He presented results of his group investigating all these epigenetic processes and how they interact. One of their most recent finding has been the observation that synovial fibroblasts differ in their phenotype depending from the localization in the body through the differential expression of miRs and lncRNAs. These findings are of fundamental importance for the homing of immune cells in health and disease.

In the following presentation, Blandine Comte (St. Genes-Champannelle, France) presented their studies on the metabolic syndrome (MetS), which relates largely to increasing obesity and sedentary lifestyle but also to early metabolic life events (not published results). In order to identify predictive biomarkers of evolution toward MetS and to bring new knowledge about this pathological state, Comte et al. used an integrative multi -omics approach in a nested case-control study. They obtained numerous results, statistically identifying 93 discriminant metabolites and 47 proteins between MetS cases and controls. Moreover, the multi -omics approach improved performance and robustness of the prediction and correlation analyses with other data contributed to better understand the role of these biomarkers in the pathological processes and therefore to evaluate their potential clinical value.

The finishing lecture of the session was given by Alexander V. Kryukov (Moscow, Russian Federation), who spoke about the apixaban, an oral nonvitamin $\mathrm{K}$ anticoagulant, which is a substrate for P-glycoprotein, encoded by ATP-binding cassette sub-family B member 1 ( $A B C B 1)$ gene and the polymorphism C3435T within this gene, which is correlated with the altered expression levels of P-glycoprotein (not published results). He postulated that the P-glycoprotein may influence the pharmacokinetic parameters of apixaban. The plasma concentration monitoring of nonvitamin $\mathrm{K}$ anticoagulants could improve safety. Thus, data about genetic factors altering pharmacokinetics of apixaban would help to develop algorithms for anticoagulant therapy personalization. The goal of this work was to determine if polymorphisms within $A B C B 1$ are associated with apixaban peak concentration in patients with acute cardioembolic stroke and atrial fibrillation. However, they did not find association between $A B C B 1$ C3435T polymorphism and apixaban peak concentration.

\section{New biomarkers and companion diagnostics}

Chairs: Lynn Bekris, Cleveland, USA/Panos Deloukas, London, UK

Starting the last of the sessions, Tomris Ozben (Antalya, Turkey) presented the potential use of the 
cell-free DNA (cfDNA) in the prognostic assessment of different solid malignancies [32]. In this study, the quality and quantity of cfDNA were assessed, furthermore cancerspecific DNA mutations as prognostic biomarkers in prostate cancer patients were tested. The results showed that patients with high cfDNA concentration at baseline had worse disease-free time and overall survival. Thus, they concluded that cfDNA detection can be used as a prognostic and predictive tool for stratification, clinical management and follow-up of prostate cancer patients.

Klaus Lindpaintner (Waltham, USA) made an introduction to the Population Resources and the requirements for Advancing Health Care [33]. The maturation of powerful technology platforms allows rapid and comprehensive mapping of genetic variants and as well as of other biomarkers, the promise of precision healthcare is becoming increasingly tangible. However, to generate the requisite information resources, a new and challenging scale of data ecosystems will be required that puts major demands on operators, users and investors. Importantly, as genetic and genomic assays are becoming commoditized, the focus is once again shifting towards detailed and sophisticated phenotyping and the exploration and inclusion of real-world data.

In his lecture, Georges Weryha (Nancy, France) was focused on osteoporosis. After many extensive programs of fundamental and clinical research, nowadays there are efficient pharmacological treatments for bone frailty. Indeed, a huge amount of clinical data demonstrates the efficiency of osteoporosis treatments [34]. GWAS and whole-exome sequencing have identified genetic determinants of monogenic and complex conditions including osteoporosis and bone mass abnormalities. The insight provided by genetic studies is serving the identification of predictive biomarkers, redefining disease, response of treatment and discovery of new therapeutic targets for skeletal disorders. Osteoporosis therapies fully belong to the new field of genomics and predictive medicine.

\section{Satellite meetings}

\section{ESPT WORKING GROUPS ON:}

\section{Pediatric individualized treatment in oncology hematology}

This group has produced a first review/position/recommendation paper (International Journal of Molecular Science) on behalf of ESPT, published in order to help clinicians to understand the role of pharmacogenomics today in pediatric oncology. In this meeting sub groups and specific recommendations/review papers have been developed.

Curriculae and education for pharmacogenomics and personalized medicine

The working group discussed about education that is necessary for the implementation of pharmacogenomics and personalized medicine, challenges and plans that can be applied.

\section{Endiobiotic and drug interactions}

The working group discussed about the necessity of clinical guidelines, of the collaboration with Drug Regulatory Agencies and the issue of translating the German Guidelines. Studies on different aspects of pharmacogenomics implementation were proposed along with economical evaluation and training.

\section{Transporter of drugs and metabolites}

The working group discussed in detail the issues of drug transporters in cancer resistance and the clinical relevance of pharmacogenetics of drug transporters as well as general aspect of pharmacogenomics of transporters in drug treatment.

\section{H2020 MSCA-RISE-2015 (MAST4HEALTH PROJECT)}

The members of the MAST4HEALTH project discussed about practical issues in NAFLD/NASH diagnosis and management and presented the interventional study with a natural product to implement safety and efficacy, which is designed by this project. The progress of the project and the next steps were presented.

\section{Round table}

The future of personalized medicine, systems medicine and systems pharmacology: how to translate the big data for the clinicians and personalized therapy.

Discussion leaders: Sofia Siest, Nancy, France/Maurizio Simmaco, Rome, Italy

At the closing of the Conference, this round table was based on all previous sessions and thematics discussed and initiated an open discussion on the challenges of translation of big data in the clinical practice. Participants agreed that this a crucial issue for the implementation of personalized medicine and identified the domains that need to be reinforced so as to overcome the weak points of the 
available strategies: education of clinicians and regulatory bodies agents, informatics tools and software that facilitate the translation of knowledge into clinical decision, translational research along with the progress of industrial products to support both research and clinical practice.

\section{Conclusions}

In conclusion, this conference raised major questions:

- Can genetic screening help identify individuals at greatest risk for cardio-metabolic diseases and cancer?

- What is the greatest clinical need with regard to diagnosis, prediction and patient stratification for these pathologies and how this is/can be addressed?

- Does a genetic risk score identify patients at highest risk and is its use justified in clinical practice?

- What are the challenges of the current clinical trials?

- What about comorbidities with aging?

- What is the impact of pharmacogenomics on:

- Deliverance of more predictable responses to drug therapy

- Minimization of the occurrence and severity of adverse drug reactions

- Conduction of more cost-effective clinical trials

- Drug discovery and the drug development process

- Do the existing diagnostic tools answer the needs of pharmacogenomics?

These questions will be addressed in the next Santorini Conference (santoriniconference.org), which will take place from 30 September to 3 October 2018.

Author contributions: All the authors have accepted responsibility for the entire content of this submitted manuscript and approved submission.

Research funding: None declared.

Employment or leadership: None declared.

Honorarium: None declared.

Competing interests: The funding organization(s) played no role in the study design; in the collection, analysis, and interpretation of data; in the writing of the report; or in the decision to submit the report for publication.

\section{References}

1. Siest G, Auffray C, Taniguchi N, Ingelman-Sundberg M, Murray H, Visvikis-Siest S, et al. Systems medicine, personalized health and therapy. Pharmacogenomics 2015;16:1527-39.
2. Siest G, Ndiaye NC, El Shamieh S, Shahabi P, Stathopoulou M, Saleh AS, et al. Conference scene: systems biology and personalized health science and translation. Pharmacogenomics 2013;14:1953-64.

3. Manolopoulos VG, Dechairo B, Huriez A, Kuhn A, Llerena A, van Schaik RH, et al. Pharmacogenomics and personalized medicine in clinical practice. Pharmacogenomics 2011;12:597-610.

4. Siest G. Systems medicine, stratified medicine, personalized medicine but not precision medicine. Drug Metabol Drug Interact 2014;29:1-2.

5. Jacobs L, Thijs L, Jin Y, Zannad F, Mebazaa A, Rouet P, et al. Heart 'omics' in AGEing (HOMAGE): design, research objectives and characteristics of the common database. J Biomed Res 2014;28:349-59.

6. Milnik A, Vogler C, Demougin P, Egli T, Freytag V, Hartmann $\mathrm{F}$, et al. Common epigenetic variation in a European population of mentally healthy young adults. J Psychiatr Res 2016;83:260-8.

7. Guo L, Milburn MV, Ryals JA, Lonergan SC, Mitchell MW, Wulff JE, et al. Plasma metabolomic profiles enhance precision medicine for volunteers of normal health. Proc Natl Acad Sci USA 2015;112:E4901-10.

8. Villoslada P, Baranzini S. Data integration and systems biology approaches for biomarker discovery: challenges and opportunities for multiple sclerosis. J Neuroimmunol 2012;248:58-65.

9. Kindler HL, Niedzwiecki D, Hollis D, Sutherland S, Schrag D, Hurwitz $\mathrm{H}$, et al. Gemcitabine plus bevacizumab compared with gemcitabine plus placebo in patients with advanced pancreatic cancer: phase III trial of the Cancer and Leukemia Group B (CALGB 80303). J Clin Oncol 2010;28:3617-22.

10. Huezo-Diaz P, Uppugunduri CR, Tyagi AK, Krajinovic M, Ansari $M$. Pharmacogenetic aspects of drug metabolizing enzymes in busulfan based conditioning prior to allogenic hematopoietic stem cell transplantation in children. Curr Drug Metab 2014;15:251-64.

11. Dutoit V, Migliorini D, Walker PR, Dietrich PY. Immunotherapy of brain tumors. Prog Tumor Res 2015;42:11-21.

12. Buchegger F, Larson SM, Mach JP, Chalandon Y, Dietrich PY, Cairoli A, et al. Radioimmunotherapy combined with maintenance anti-CD20 antibody may trigger long-term protective $T$ cell immunity in follicular lymphoma patients. Clin Dev Immunol 2013;2013:875343.

13. Fouquet G, Tardy S, Demarquette H, Bonnet S, Gay J, Debarri H, et al. Efficacy and safety profile of long-term exposure to lenalidomide in patients with recurrent multiple myeloma. Cancer 2013;119:3680-6.

14. Ansari M, Krajinovic M. Pharmacogenomics of acute leukemia. Pharmacogenomics 2007;8:817-34.

15. Bekris LM, Lutz F, Yu CE. Functional analysis of APOE locus genetic variation implicates regional enhancers in the regulation of both TOMM40 and APOE. J Hum Genet 2012;57:18-25.

16. Bekris LM, Millard SP, Galloway NM, Vuletic S, Albers JJ, Li G, et al. Multiple SNPs within and surrounding the apolipoprotein E gene influence cerebrospinal fluid apolipoprotein E protein levels. J Alzheimers Dis 2008;13:255-66.

17. Buntru A, Trepte P, Klockmeier K, Schnoegl S, Wanker EE. Current approaches toward quantitative mapping of the interactome. Front Genet 2016;7:74.

18. Hosp F, Vossfeldt H, Heinig M, Vasiljevic D, Arumughan A, Wyler E, et al. Quantitative interaction proteomics 
of neurodegenerative disease proteins. Cell Rep 2015;11: 1134-46.

19. Umlauf E, Rappold E, Schiller B, Fuchs P, Rainer M, Wolf B, et al. Careful neuropsychological testing reveals a novel genetic marker, GSTO ${ }^{*} \mathrm{C}$, linked to the pre-stage of Alzheimer's disease. Oncotarget 2016;7:39108-17.

20. Sudlow C, Gallacher J, Allen N, Beral V, Burton P, Danesh J, et al. UK biobank: an open access resource for identifying the causes of a wide range of complex diseases of middle and old age. PLoS Med 2015;12:e1001779.

21. Buck Louis GM, Smarr MM, Patel CJ. The exposome research paradigm: an opportunity to understand the environmental basis for human health and disease. Curr Environ Health Rep 2017;4:89-98.

22. Petta S, Gastaldelli A, Rebelos E, Bugianesi E, Messa P, Miele L, et al. Pathophysiology of non alcoholic fatty liver disease. Int J Mol Sci 2016;17:2082.

23. Trabado S, Al-Salameh A, Croixmarie V, Masson P, Corruble E, Feve B, et al. The human plasma-metabolome: reference values in 800 French healthy volunteers; impact of cholesterol, gender and age. PLoS One 2017;12:e0173615.

24. El-Sayed Moustafa JS, Froguel P. From obesity genetics to the future of personalized obesity therapy. Nat Rev Endocrinol 2013;9:402-13.

25. Kaliora AC, Kokkinos A, Diolintzi A, Stoupaki M, Gioxari A, Kanellos PT, et al. The effect of minimal dietary changes with raisins in NAFLD patients with non-significant fibrosis: a randomized controlled intervention. Food Funct 2016;7:4533-44.

26. Botticelli A, Onesti CE, Strigari L, Occhipinti M, Di Pietro FR, Cerbelli B, et al. A nomogram to predict 5-fluorouracil toxicity: when pharmacogenomics meets the patient. Anticancer Drugs 2017;28:551-6.
27. Nofziger C, Papaluca M, Terzic A, Waldman S, Paulmichl M. Policies to aid the adoption of personalized medicine. Nat Rev Drug Discov 2014;13:159-60.

28. Pratt VM, Everts RE, Aggarwal P, Beyer BN, Broeckel U, EpsteinBaak R, et al. Characterization of 137 genomic DNA reference materials for 28 pharmacogenetic genes: a GeT-RM collaborative project. J Mol Diagn 2016;18:109-23.

29. ten Brink MH, van Bavel T, Swen JJ, van der Straaten T, Bredius RG, Lankester AC, et al. Effect of genetic variants GSTA1 and CYP39A1 and age on busulfan clearance in pediatric patients undergoing hematopoietic stem cell transplantation. Pharmacogenomics 2013;14:1683-90.

30. Francino MP. Early development of the gut microbiota and immune health. Pathogens 2014;3:769-90.

31. Ospelt C, Gay S, Klein K. Epigenetics in the pathogenesis of RA. Semin Immunopathol 2017. doi: 10.1007/s00281-0170621-5.

32. Miyamoto DT, Lee RJ. Cell-free and circulating tumor cell-based biomarkers in men with metastatic prostate cancer: tools for real-time precision medicine? Urol Oncol 2016;34:490-501.

33. Harris JR, Burton P, Knoppers BM, Lindpaintner K, Bledsoe M, Brookes AJ, et al. Toward a roadmap in global biobanking for health. Eur J Hum Genet 2012;20:1105-11.

34. Diedhiou D, Cuny T, Sarr A, Norou Diop S, Klein M, Weryha G. Efficacy and safety of denosumab for the treatment of osteoporosis: a systematic review. Ann Endocrinol (Paris) 2015;76:650-7.

Supplemental Material: The online version of this article (DOI: 10.1515/dmpt-2017-0011) offers supplementary material, available to authorized users. 